\title{
Ultrassonografia uretrovesical como método eficaz de avaliação dos parâmetros prognósticos de tratamento cirúrgico da incontinência urinária de esforço*
}

\author{
Urethrovesical ultrasonography as an effective method for evaluating prognostic \\ parameters in the surgical management of stress urinary incontinence \\ Frederico Teixeira Brandt ${ }^{1}$, Risomar Belarmino Almeida ${ }^{2}$, Arinaldo Vasconcelos de Alencar ${ }^{3}$, \\ Carla Daisy Costa Albuquerque ${ }^{3}$, Agostinho de Sousa Machado Junior ${ }^{4}$
}

Resumo OBJETIVO: Comparar parâmetros ultrassonográficos relacionados à junção uretrovesical e uretra proximal em pacientes curadas e não curadas, tratadas cirurgicamente pela técnica de Burch, com a finalidade de estabelecer se há correspondência com fatores prognósticos. MATERIAIS E MÉTODOS: Trinta pacientes foram selecionadas e divididas em dois grupos: 15 consideradas clinicamente curadas e 15 consideradas não curadas. As pacientes foram submetidas a ultrassonografia transvulvar no pré-operatório e aos 30 e 180 dias do pós-operatório. RESULTADOS: No pré-operatório, o deslocamento da uretra proximal foi maior nas pacientes curadas $(15,87 \pm 4,55 \mathrm{~mm} \times 12,47 \pm 3,52 \mathrm{~mm}-p<0,05)$. No pós-operatório, no esforço, a distância vertical da junção uretrovesical e a uretra proximal foram maiores nas pacientes curadas $(12,87 \pm$ $5,80 \mathrm{~mm} \times 5,13 \pm 6,55 \mathrm{~mm}-p<0,01$; e 13,07 $\pm 6,44 \mathrm{~mm} \times 6,20 \pm 6,14 \mathrm{~mm}-p<0,01)$, e o deslocamento vertical da junção uretrovesical e da uretra proximal foi maior nas pacientes não curadas $(8,47 \pm 3,98 \mathrm{~mm} \times 5,13 \pm 2,36 \mathrm{~mm}-p<0,001$; e 8,33 $\pm 4,54 \mathrm{~mm} \times 5,20 \pm 2,90 \mathrm{~mm}-p<0,05)$. CONCLUSÃO: A ultrassonografia da junção uretrovesical e da uretra proximal pode ser considerada como um método eficaz de avaliação dos parâmetros prognósticos do tratamento cirúrgico de mulheres com incontinência urinária de esforço.

Unitermos: Ultrassonografia transvulvar; Ultrassonografia perineal; Junção uretrovesical; Incontinência urinária de esforço; Cirurgia de Burch.

Abstract OBJECTIVE: To compare sonographic parameters related to the urethrovesical junction and proximal urethra in cured and not cured patients surgically treated with the Burch technique, for establishing a possible correlation with prognostic factors. MATERIALS AND METHODS: Thirty female patients were selected and divided into two groups: 15 patients considered as clinically cured and 15 not cured. The patients were submitted to preoperative transvulvar ultrasonography, with the examination being repeated at the 30th and 180th postoperative days. RESULTS: At the preoperative examination, the proximal urethra displacement was largest in the cured patients $(15.87 \pm 4.55 \mathrm{~mm} \times 12.47 \pm 3.52 \mathrm{~mm}-p<0.05)$. At the postoperative examination, with strain, the vertical distance of the urethrovesical junction and the proximal urethra were largest in the cured patients $(12.87 \pm 5.80 \mathrm{~mm} \times 5.13 \pm 6.55 \mathrm{~mm}-p<0.01$; and $13.07 \pm 6.44 \mathrm{~mm}$ $\times 6.20 \pm 6.14 \mathrm{~mm}-p<0.01$ ), and the vertical displacement of the urethrovesical junction and proximal urethra was largest in the not cured patients $18.47 \pm 3.98 \mathrm{~mm} \times 5.13 \pm 2.36 \mathrm{~mm}-p<0.001$; and 8.33 $\pm 4.54 \mathrm{~mm} \times 5.20 \pm 2.90 \mathrm{~mm}-p<0.05)$. CONCLUSION: Ultrasonography of the urethrovesical junction and proximal urethra can be considered as an effective method for evaluating prognostic parameters in the surgical management of women with stress urinary incontinence.

Keywords: Transvulvar ultrasonography; Perineal ultrasonography; Urethrovesical junction; Stress urinary incontinence; Burch surgery.

Brandt FT, Almeida RB, Alencar AV, Albuquerque CDC, Machado Jr AS. Ultrassonografia uretrovesical como método eficaz de avaliação dos parâmetros prognósticos de tratamento cirúrgico da incontinência urinária de esforço. Radiol Bras. 2009;42(3):165-169.

* Trabalho realizado na Unidade de Pesquisa em Incontinência Urinária da Universidade Federal de Pernambuco (UFPE), Recife, PE, Brasil.

1. Livre-Docente, Professor da Universidade Federal de Pernambuco (UFPE), Recife, PE, Brasil.

2. Mestre, Médica da Unidade de Pesquisa em Incontinência Urinária da Universidade Federal de Pernambuco (UFPE), Recife, PE, Brasil.

3. Doutores, Professores Adjuntos da Universidade Federal de Pernambuco (UFPE), Recife, PE, Brasil.
4. Residência Médica em Ginecologia e Obstetrícia, Professor Substituto de Obstetrícia da Universidade Federal de Pernambuco (UFPE), Recife, PE, Brasil.

Endereço para correspondência: Dr. Frederico Teixeira Brandt. Avenida Dezessete de Agosto, 2475, ap. 2801, Monteiro. Recife, PE, Brasil, 52061-540. E-mail: fcbrandt@elogica. com.br

Recebido para publicação em 22/11/2008. Aceito, após revisão, em 5/3/2009.

\section{INTRODUÇÃO}

A incontinência urinária de esforço (IUE) se reveste de grande importância, não somente como doença, mas, principalmente, pelas repercussões sociais e pela forma como altera a qualidade de vida das 
pacientes. Em razão da sua alta prevalência, são despendidos, anualmente, em torno de 26 bilhões de dólares nos Estados Unidos, sendo cerca de 393,5 milhões em testes diagnósticos ${ }^{(1-3)}$.

O diagnóstico pressuposto da IUE é eminentemente clínico. Para se confirmar a hipótese clínica, o exame complementar mais difundido é a avaliação urodinâmica da bexiga e da uretra, que mostra a perda de urina na ausência de contração do músculo detrusor. Entretanto, a avaliação urodinâmica determina apenas de forma indireta a mobilidade vertical da junção uretrovesical (JUV) e da uretra proximal (UP). Diferentemente, a ultrassonografia mostra imagem real, posicionamento e mobilidade da JUV e da UP(4-20).

O exame ultrassonográfico da JUV e da UP tem sido usado cada vez mais na avaliação pré-operatória de pacientes com IUE e no pós-operatório, para estabelecer parâmetros topográficos de posicionamento da JUV e da UP em conformidade com a técnica cirúrgica utilizada ${ }^{(4-13,17-19)}$. Estudo inédito mostra que o posicionamento da JUV e da UP no pós-operatório é um bom fator prognóstico do sucesso ou insucesso da correção cirúrgica da IUE ${ }^{(14)}$.

Pretende-se, neste estudo, a partir da cirurgia de Burch, tendo a ultrassonografia transvulvar como método de investigação de pacientes com IUE, analisar o posicionamento e a mobilidade da JUV e da UP como fator prognóstico.

\section{MATERIAIS E MÉTODOS}

O projeto foi aprovado pelo Conselho de Ética do Centro de Ciências da Saúde da Universidade Federal de Pernambuco (UFPE). Foram selecionadas 30 mulheres, submetidas à cirurgia de Burch para tratamento de IUE, no período de janeiro de 2005 a agosto de 2007, e que procuraram a Unidade de Pesquisa em Incontinência Urinária da UFPE para avaliação pós-operatória. Essas 30 mulheres foram divididas, em relação à queixa principal de IUE, em dois grupos: grupo $1-15$ pacientes que referiram cura; grupo $2-15$ pacientes que voltaram a ter a mesma queixa que motivou a cirurgia.

Em cada paciente investigada foi realizado exame ultrassonográfico com até 30 dias do pré-operatório, assim como no controle do pós-operatório. A ultrassonografia transvulvar da JUV e da UP foi realizada com aparelho da marca Aloka SSD 500, após a paciente urinar (bexiga com menos de $50 \mathrm{ml}$ de urina). A paciente assumia a posição ginecológica e um transdutor vaginal convexo, com frequência de $7 \mathrm{MHz}$, revestido com condom estéril, era suavemente colocado entre os grandes lábios, com a pressão necessária para obtenção das imagens, mas sem exercer qualquer compressão que comprometesse o deslocamento das estruturas a investigar. Foram obtidas imagens do púbis, da uretra, da JUV e da bexiga, com a finalidade de aferir a distância pubouretral (DPU), a distância vertical da JUV (DVJUV), a distância horizontal da JUV (DHJUV) e a UP, em repouso. A seguir, era então solicitado que a paciente fizesse o máximo de esforço físico abdominal para baixo (manobra de Valsalva) e as mesmas medidas eram obtidas. Neste trabalho foram avaliadas a DPU, a DHJUV, a DVJUV, a UP e variações dos seus deslocamentos.

As variáveis ultrassonográficas são assim conceituadas:

DPU - comprimento, em milímetros, de uma linha horizontal traçada da borda inferior da sínfise púbica à porção uretral que atravessa essa linha. $\mathrm{O}$ cruzamento da linha horizontal com a porção da uretra que a atravessa recebe o nome de ponto uretral;

UP - distância, em milímetros, da JUV ao ponto uretral, correspondendo à UP;

DHJUV - comprimento, em milímetros, de uma linha horizontal, iniciada na JUV, até o encontro com uma linha vertical originada na borda inferior da sínfise púbica. Os valores à esquerda da linha são positivos e à direita são negativos;

DVJUV - comprimento, em milímetros, de uma linha vertical traçada da JUV até o encontro da linha horizontal que passa pela borda inferior da sínfise púbica. Os valores acima dessa linha horizontal são positivos e os valores abaixo são negativos;

Mobilidade horizontal da JUV - distância horizontal, em milímetros, percorrida pela JUV durante o esforço (manobra de Valsalva). É a diferença entre a DHJUV no repouso para a DHJUV no esforço;

Mobilidade vertical da JUV - distância vertical, em milímetros, percorrida pela JUV durante o esforço (manobra de Valsalva). É a diferença entre a DVJUV no repouso para a DVJUV no esforço;

Variação da DPU - é a diferença existente entre a DPU no repouso e a DPU no esforço;

Variação da UP - é a diferença entre a UP no repouso e a UP no esforço.

Os parâmetros ultrassonográficos seguiram os conceitos já especificados, ilustrados nas Figuras 1 e 2.

A cirurgia combinada de Burch era realizada executando-se incisão de Pfannenstiel, e no espaço de Retzius se identificavam a JUV, a uretra, a fáscia pubocervical do terço inferior da vagina e a junção vesicovaginal. Dois pontos com fio prolene zero eram aplicados na fáscia parauretral direita, distando $2 \mathrm{~cm} \mathrm{e} 4 \mathrm{~cm}$ da uretra e a $1 \mathrm{~cm}$ da base vesical. Os mesmos pontos eram aplicados no lado esquerdo da uretra. Os fios transfixavam os ligamentos de Cooper bilateralmente, mantendo orientação paralela entre eles, e eram amarrados com moderada tensão para elevar a fáscia parauretral e a JUV.

Os resultados das variáveis contínuas foram expressos por suas médias e desvios-padrão da média. Para cada uma das variáveis estudada nos diferentes grupos foi feita a análise de variância. Após cada análise, a comparação dos resultados do grupo 1 com os resultados do grupo 2 foi feita por meio do teste de Fisher. Foi aceito o intervalo de confiança de $95 \%$ e o nível de significância foi considerado para valor de $p$ menor que $0,05(p<0,05)$.

\section{RESULTADOS}

Os resultados estão apresentados nas Tabelas 1 a 4, e descritos a seguir.

A DPU, em condições de repouso e esforço, não variou significativamente $(p>$ $0,05)$ entre os grupos 1 e 2 , nos períodos pré- e pós-operatório (Tabela 1).

A DHJUV não variou significativamente $(p>0,05)$ entre os grupos 1 e 2 , nas fases de pré- e pós-operatório, nas condições de repouso, esforço e deslocamento (Tabela 2).

No período pós-operatório, na condição de esforço, observa-se que no grupo 2 a DVJUV foi significativamente menor e o deslocamento da JUV, significativamente maior (Tabela 3). 


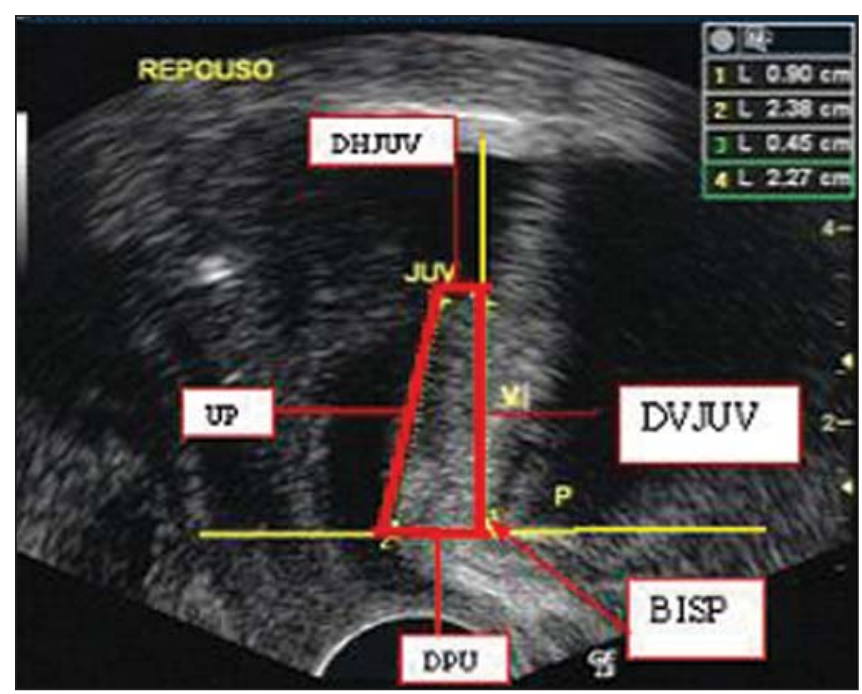

Figura 1. Imagem ultrassonográfica transvulvar mostrando bexiga, UP e púbis, orientando a forma de obtenção das variáveis DPU, UP, DHJUV e DVJUV. Imagem obtida com a paciente em repouso.

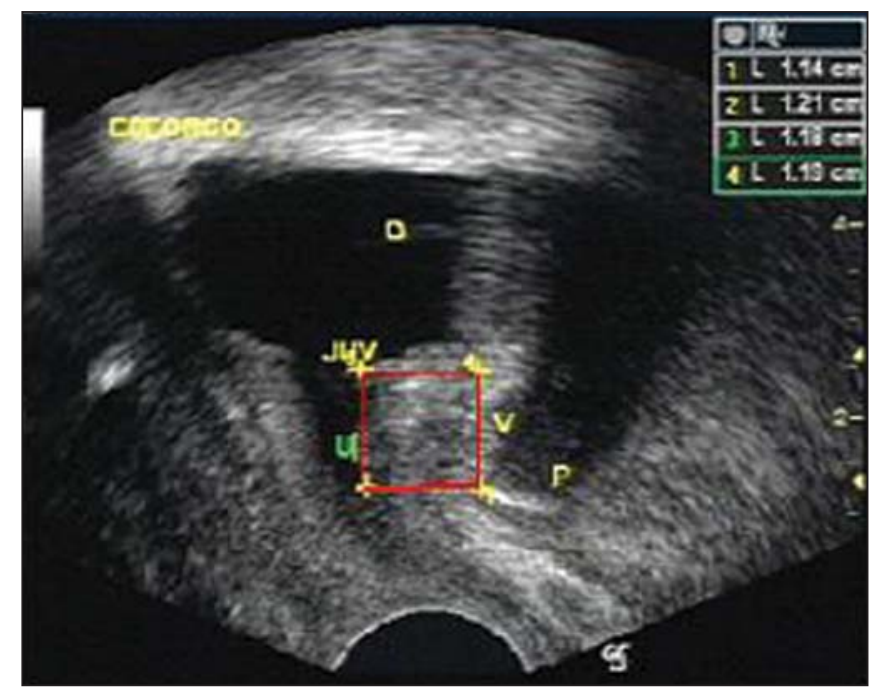

Figura 2. Imagem ultrassonográfica transvulvar mostrando bexiga, UP e púbis, orientando a forma de obtenção das variáveis DPU, UP, DHJUV e DVJUV. Imagem obtida com a paciente em esforço.

Tabela 1 Valores das médias e desvios-padrão (em milímetros) e níveis de significância da variável DPU nas fases de pré- e pós-operatório, em condições de repouso, esforço e deslocamento, das mulheres curadas (grupo 1) e não curadas (grupo 2).

\begin{tabular}{|c|c|c|c|c|c|c|}
\hline \multirow[b]{2}{*}{ Especificação } & \multicolumn{3}{|c|}{ Pré-operatório } & \multicolumn{3}{|c|}{ Pós-operatório } \\
\hline & Repouso & Esforço & Deslocamento & Repouso & Esforço & Deslocamento \\
\hline Média grupo 1 & 13,73 & 21,67 & 7,93 & 11,47 & 10,40 & $-1,07$ \\
\hline Média grupo 2 & 13,47 & 18,13 & 4,80 & 11,80 & 11,27 & $-0,53$ \\
\hline Desvio-padrão grupo 1 & 4,23 & 7,93 & 5,62 & 3,04 & 3,77 & 3,97 \\
\hline Desvio-padrão grupo 2 & 3,07 & 4,98 & 3,78 & 4,09 & 4,82 & 5,19 \\
\hline Valor de $p$ & $p>0,05^{\mathrm{ns}}$ & $p>0,05^{\mathrm{ns}}$ & $p>0,05^{\mathrm{ns}}$ & $p>0,05^{\mathrm{ns}}$ & $p>0,05^{\mathrm{ns}}$ & $p>0,05^{\mathrm{ns}}$ \\
\hline
\end{tabular}

ns Não significativo, pelo teste de Fisher, para comparação de variâncias.

Tabela 2 Valores das médias e desvios-padrão (em milímetros) e níveis de significância da variável DHJUV nas fases de pré- e pós-operatório, em condições de repouso, esforço e deslocamento, das mulheres curadas (grupo 1) e não curadas (grupo 2).

\begin{tabular}{|c|c|c|c|c|c|c|}
\hline \multirow[b]{2}{*}{ Especificação } & \multicolumn{3}{|c|}{ Pré-operatório } & \multicolumn{3}{|c|}{ Pós-operatório } \\
\hline & Repouso & Esforço & Deslocamento & Repouso & Esforço & Deslocamento \\
\hline Média grupo 1 & 15,27 & 21,73 & 6,87 & 5,87 & 6,20 & 0,33 \\
\hline Média grupo 2 & 13,47 & 18,87 & 6,60 & 8,47 & 9,40 & 0,93 \\
\hline Desvio-padrão grupo 1 & 7,17 & 7,61 & 4,49 & 5,57 & 5,76 & 3,39 \\
\hline Desvio-padrão grupo 2 & 6,62 & 5,71 & 6,28 & 5,74 & 6,16 & 7,10 \\
\hline Valor de $p$ & $p>0,05^{\mathrm{ns}}$ & $p>0,05^{\text {ns }}$ & $p>0,05^{\mathrm{ns}}$ & $p>0,05^{\mathrm{ns}}$ & $p>0,05^{\mathrm{ns}}$ & $p>0,05^{\mathrm{ns}}$ \\
\hline
\end{tabular}

ns Não significativo, pelo teste de Fisher, para comparação de variâncias.

Tabela 3 Valores das médias e desvios-padrão (em milímetros) e níveis de significância da variável DVJUV nas fases de pré- e pós-operatório, em condições de repouso, esforço e deslocamento, das mulheres curadas (grupo 1) e não curadas (grupo 2).

\begin{tabular}{|c|c|c|c|c|c|c|}
\hline \multirow[b]{2}{*}{ Especificação } & \multicolumn{3}{|c|}{ Pré-operatório } & \multicolumn{3}{|c|}{ Pós-operatório } \\
\hline & Repouso & Esforço & Deslocamento & Repouso & Esforço & Deslocamento \\
\hline Média grupo 1 & 17,20 & 4,27 & 17,20 & 17,20 & 12,87 & 5,13 \\
\hline Média grupo 2 & 15,40 & 5,20 & 14,07 & 13,60 & 5,13 & 8,47 \\
\hline Desvio-padrão grupo 1 & 5,68 & 4,06 & 5,21 & 4,96 & 5,80 & 2,36 \\
\hline Desvio-padrão grupo 2 & 4,63 & 4,74 & 4,93 & 6,29 & 6,55 & 3,98 \\
\hline Valor de $p$ & $p>0,05^{\text {ns }}$ & $p>0,05^{\text {ns }}$ & $p>0,05^{\text {ns }}$ & $p>0,05^{\mathrm{ns}}$ & $p<0,01^{*}$ & $p<0,01^{*}$ \\
\hline
\end{tabular}

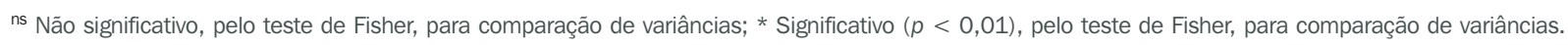


Tabela 4 Valores das médias e desvios-padrão (em milímetros) e níveis de significância da variável comprimento da UP nas fases de pré- e pós-operatório, em condições de repouso, esforço e deslocamento, das mulheres curadas (grupo 1) e não curadas (grupo 2).

\begin{tabular}{|c|c|c|c|c|c|c|}
\hline \multirow[b]{2}{*}{ Especificação } & \multicolumn{3}{|c|}{ Pré-operatório } & \multicolumn{3}{|c|}{ Pós-operatório } \\
\hline & Repouso & Esforço & Deslocamento & Repouso & Esforço & Deslocamento \\
\hline Média grupo 1 & 17,87 & 2,00 & 15,87 & 18,27 & 13,07 & 5,20 \\
\hline Média grupo 2 & 15,93 & 3,47 & 12,47 & 14,53 & 6,20 & 8,33 \\
\hline Desvio-padrão grupo 1 & 5,66 & 3,61 & 4,55 & 5,60 & 6,44 & 2,90 \\
\hline Desvio-padrão grupo 2 & 4,46 & 5,41 & 3,52 & 7,04 & 6,14 & 4,54 \\
\hline Valor de $p$ & $p>0,05^{\text {ns }}$ & $p>0,05^{\text {ns }}$ & $p<0,05^{*}$ & $p>0,05^{\mathrm{ns}}$ & $p<0,01^{\dagger}$ & $p<0,05^{*}$ \\
\hline
\end{tabular}

ns Não significativo, pelo teste de Fisher, para comparação de variâncias; * Significativo $(p<0,05)$, pelo teste de Fisher, para comparação de variâncias; ${ }^{\dagger}$ Significativo $(p<0,01)$, pelo teste de Fisher, para comparação de variâncias.

Com relação à variável UP, houve diferença significativa $(p<0,05)$ entre os grupos 1 e 2 para a condição de deslocamento, tanto no pré- como no pós-operatório, e na condição de esforço no pós-operatório $(p$ $<0,01)$. Os menores desvios-padrão foram observados na condição de deslocamento, sendo o do grupo 1 observado no pós-operatório $(2,90 \mathrm{~mm})$ e o do grupo 2 , no pré-operatório (3,52 mm) (Tabela 4).

\section{DISCUSSÃO}

No presente estudo, o objetivo principal foi testar a hipótese já publicada na literatura de que a investigação ultrassonográfica transvulvar da JUV e da UP serve como exame de avaliação prognóstica do tratamento cirúrgico da $\mathrm{IUE}^{(\mathbf{1 4})}$.

$\mathrm{O}$ grande diferencial de nossa pesquisa é a demonstração inédita de que o fator prognóstico já pode ser detectado no pré-operatório, por meio de um menor deslocamento da UP em mulheres com recidiva de IUE. Mas, nossos resultados precisam ser testados por outros pesquisadores. É nossa pretensão investigar a mesma hipótese em outras cirurgias indicadas para tratamento de IUE.

Para responder à pergunta formulada, foi utilizada a cirurgia de Burch porque a referida técnica pode ser considerada padrão ouro ${ }^{(18)}$ e já é realizada de forma padronizada na Unidade de Pesquisa em Incontinência Urinária da UFPE por mais de dez anos. Porém, até que se prove o contrário, pelo argumento de que o tratamento cirúrgico deve reposicionar a JUV e a UP em condições compatíveis com a lógica da continência urinária passiva, o que é válido para a cirurgia de Burch deve corresponder a outro procedimento cirúrgico ${ }^{(15)}$.
Baseado no conceito de continência urinária passiva, a correção cirúrgica da IUE consiste em refazer de forma permanente a mobilidade vertical da JUV e o comprimento da $U P^{(16)}$. Assim, foi observado na presente pesquisa que no pós-operatório das pacientes curadas apenas uma delas $(6,7 \%)$ apresentou hipermobilidade, enquanto no grupo das pacientes não curadas esse percentual elevou-se para 53,4\%.

Embora a pesquisa tenha sido realizada em mulheres com IUE tratadas pela cirurgia de Burch, teoricamente é possível extrapolar que o mesmo comportamento anatomofisiológico deve ocorrer com qualquer outra técnica para tratar IUE. Portanto, a avaliação ultrassonográfica da JUV e da UP deve ser adequada, no pré- e pós-operatório de uma paciente tratada de IUE por outro procedimento cirúrgico, para determinar os fatores prognósticos de cura ou não cura.

Ainda não se pode afirmar qual a importância real do posicionamento horizontal da JUV e da UP na investigação de IUE no prée pós-operatório. É bem provável que haja alguma correlação que não está esclarecida devidamente. Mas, é possível afirmar que há correlação entre esses parâmetros ultrassonográficos, avaliados de forma estática e dinâmica, com o sucesso e insucesso do tratamento cirúrgico de mulheres com IUE.

\section{CONCLUSÃO}

A ultrassonografia da JUV e da UP pode ser considerada como método eficaz de avaliação dos parâmetros prognósticos do tratamento cirúrgico de mulheres com IUE.

\section{REFERÊNCIAS}

1. Fultz N, Girts T, Kinchen K, et al. Prevalence, management and impact of urinary incontinence in the workplace. Occup Med (Lond). 2005;55: 552-7.

2. Weber AM, Taylor RJ, Wei JT, et al. The cost-effectiveness of preoperative testing (basic office assessment versus urodynamics) for stress urinary incontinence in women. BJU Int. 2002;89:35663.

3. Kocak I, Okyay P, Dundar M, et al. Female urinary incontinence in the west of Turkey: prevalence, risk factors and impact on quality of life. Eur Urol. 2005;48:634-41.

4. Brandt FT, Albuquerque CDC, Lorenzato FR, et al. Perineal assessment of urethrovesical junction mobility in young continent females. Int Urogynecol J Pelvic Floor Dysfunct. 2000;11:18-22.

5. Brandt FT, Oliveira RR, Albuquerque CDC, et al. Incontinência urinária de urgência no contexto da incontinência urinária de esforço. J Bras Ginecol. 1998;98:183-4.

6. Brandt FT, Albuquerque CDC, Arraes AF, et al. Influência do volume vesical na avaliação ultrasonográfica da junção uretrovesical e uretra proximal. Radiol Bras. 2005;38:33-6.

7. Tunn R, Petri E. Introital and transvaginal ultrasound as the main tool in the assessment of urogenital and pelvic floor dysfunction: an imaging panel and practical approach. Ultrasound Obstet Gynecol. 2003;22:205-13.

8. Brandt FT, Nóbrega LV, Albuquerque CDC, et al. Aferição simultânea da pressão abdominal na avaliação ultra-sonográfica de mulheres com incontinência urinária de esforço. Radiol Bras. 2006;39:91-5.

9. Brandt FT, Santos Junior MW, Albuquerque CDC, et al. Modificações da junção uretrovesical e uretra proximal após a cirurgia de alça sem tensão em mulheres de 45 a 72 anos. Rev Bras Saude Mater Infant. 2005;5:185-91.

10. Dalpiaz O, Curti P. Role of perineal ultrasound in the evaluation of urinary stress incontinence and pelvic organ prolapse: a systematic review. Neurourol Urodyn. 2006;25:301-6.

11. Sendag F, Vidinli H, Kazandi M, et al. Role of perineal sonography in the evaluation of patients with stress urinary incontinence. Aust N Z J Obstet Gynaecol. 2003;43:54-7.

12. Weidner AC, Myers ER, Visco AG, et al. Which women with stress incontinence require urodynamic evaluation? Am J Obstet Gynecol. 2001; 184:20-7.

13. Kil PJ, Hoekstra JW, van der Meijden AP, et al. Transvaginal ultrasonography and urodynamic evaluation after suspension operations: compari- 
son among the Gittes, Stamey and Burch suspensions. J Urol. 1991;146:132-6.

14. Viereck V, Pauer HU, Hesse O. Urethral hypermobility after anti-incontinence surgery - a prognostic indicator? Int Urogynecol J Pelvic Floor Dysfunct. 2006; 17:586-92.

15. Ribeiro CBL, Brandt FT, Albuquerque CD, et al Modificações da uretra proximal e da junção uretrovesical, decorrentes da cirurgia do tipo KellyKennedy. Acta Cir Bras. 2001;17:21-3.

16. Brandt FT. Importância da junção uretrovesical na continência urinária passiva e na incontinên- cia urinária de esforço. J Bras Ginecol. 1996;106: 259-62.

17. Brandt FT, Albuquerque CDC, Lorenzato FRB, et al. A importância da ultra-sonografia transvulvar na avaliação de parâmetros anatômicos relevantes no tratamento de mulheres com incontinência urinária de esforço. Radiol Bras. 2007;40: 371-6.

18. Martan A, Masata J, Halaska M, et al. Ultrasound imaging of the lower urinary system in women after Burch colposuspension. Ultrasound Obstet Gynecol. 2001;17:58-64.
19. Chene G, Cotte B, Tardieu AS, et al. Clinical and ultrasonographic correlations following three surgical anti-incontinence procedures (TOT, TVT and TVT-O). Int Urogynecol J Pelvic Floor Dysfunct. 2008;19:1125-31.

20. Brandt FT, Lorenzato F, Albuquerque CDC, et al Tension-free vaginal tape versus lata fascia sling: the importance of transvulvar ultrasound in the assessment of relevant anatomical parameters in treatment of women with stress urinary incontinence. Indian J Urol. 2009;25:62-7. 